\title{
A Dual-Frequency Patch Antenna with Monopolar Radiation Pattern
}

\author{
Francisco Javier Herraiz-Martínez*(1), Eduardo Ugarte-Muñoz ${ }^{(1)}$, Vicente \\ González-Posadas $^{(2)}$, and Daniel Segovia-Vargas ${ }^{(1)}$ \\ (1) Department of Signal Theory and Communications, Carlos III University in \\ Madrid, Leganés, Madrid, Spain, 28911 \\ (2) Departamento de Ingeniería Audiovisual y Comunicationes, Universidad \\ Politécnica de Madrid, Madrid, Spain \\ E-mail: fjherraiz@tsc.uc3m.es
}

\section{Introduction}

Concentrically shorted patch microstrip antennas have the unique feature that their fundamental mode has a monopolar-like radiation pattern (omnidirectional with $\mathrm{E}_{\theta}$ polarization and the maximum in the horizon) [1-2]. This is contrary to conventional patch antennas whose fundamental mode has a broadside radiation pattern. Initially the fundamental monopolar mode was achieved with circular geometries [2], but later it was demonstrated that can be excited in non-circular patches shorted in the center [3]. More recently, great efforts have been made on achieving miniaturized patch antennas with the monopolar radiation pattern. Good results have been reported by using patches composed of metamaterial cells [4-5].

During the nineties, this type of patch antennas was extensively used in the field of user terminals for cellular communications [3]. After that, monopolar patch antennas have been applied to WLANs (Wireless Local Area Networks). Multifrequency patch antennas with monopolar radiation pattern could be a very interesting radiating element for access points and user terminals due to the increase of standards and bands allowed for wireless communications and WLANs. However, up to the authors' knowledge, monopolar patch antennas with multi-frequency performance have not been reported yet. In this communication, a patch antenna with two monopolar modes is proposed. These monopolar modes can be excited by using one or two different coaxial ports. The second approach is chosen in this design, achieving a high isolation between the ports $(>10 \mathrm{~dB})$.

\section{Antenna Design and Results}

The conventional approach to achieve a microstrip patch antenna with a fundamental monopolar mode consists of inserting a shorting metallic cylinder in the centre of the patch [1-3]. Similar results can be achieved by using shorting vias placed in the proper locations [6]. The advantage of this second approach is that it is easier to manufacture. In both cases, the fundamental mode has an electric-field distribution with uniform amplitude and phase. Therefore, the equivalent magnetic current densities form a loop that produces the monopolar radiation pattern. The proposed approach consists of inserting two concentric 
short conditions in a conventional patch antenna with a separating gap between them. Hence, the equivalent current densities at the edges depend on the electric field at the outer part of the patch. Equivalently, the radiation pattern is independent of the "radial" electric-field distribution, but depends only on the distribution at the edges. Two different modes with a uniform electric-field distribution at the outer part of the patch are achieved and thus, a monopolar radiation pattern is obtained at two different frequencies.

Fig. 1 shows the sketch of the proposed antenna. It is based on a square patch antenna with a gap to separate the two shorts. Each shorting condition is implemented with four vias placed at the same distance from the centre of the patch. The outer vias are placed next to the gap. The parameters of the antenna are the length of the square patch $(L)$, the position and the width of the gap $\left(d_{g a p}\right.$ and $w_{\text {gap }}$, respectively), the diameter of the shorting vias $\left(d_{\text {vias }}\right)$, the position of the inner vias $\left(d_{\text {short }}\right)$ and the permittivity and thickness of the dielectric substrate $(h$ and $\varepsilon_{\mathrm{r}}$, respectively). The working frequencies, the ratio between them and the input impedance depend manly on the length of the patch $(L)$ and the position of the shorts $\left(d_{g a p}\right.$ and $\left.d_{\text {short }}\right)$. Two different feeding strategies have been studied. The first one consists of using one coaxial port which excites the two working modes. The other one is based on two different coaxial ports, each of them exciting a different monopolar mode. It is possible two achieve simultaneous matching at the two monopolar frequencies with both feeding approaches. The second approach has been chosen for the proposed example (Fig. 1). It is important to note that good isolation between both ports is achieved, as it will be shown below.

The dimensions of the proposed patch are: $L=31.00 \mathrm{~mm}, d_{\text {gap }}=7.50 \mathrm{~mm}, w_{\text {gap }}=$ $0.40 \mathrm{~mm}, d_{\text {vias }}=0.70 \mathrm{~mm}$ and $d_{\text {short }}=3.75 \mathrm{~mm}$. The dielectric substrate is polypropylene with $\varepsilon_{\mathrm{r}}=2.2$ and $h=2.00 \mathrm{~mm}$. The distances between the ports and the centre of the patch are $9.00 \mathrm{~mm}$ for the port $\# 1$ and $6.00 \mathrm{~mm}$ for the port \#2. This antenna has been simulated with CST Microwave Studio ${ }^{\circledR}$. Fig. 2 shows the simulated [S] parameters. The reflection coefficient at each port is completely different. The first resonance at port \#1 occurs at $2.6 \mathrm{GHz}$, while the first resonance at port \#2 appears at $4.8 \mathrm{GHz}$. Good matching is obtained at both frequencies. The isolation between both ports is higher than $10 \mathrm{~dB}$ at the first frequency and $22 \mathrm{~dB}$ at the second one. The second resonance appearing at port $\# 1(3.4 \mathrm{GHz})$ is the conventional patch-like $\mathrm{TM}_{10}$ mode.

The electric-field distributions at the two frequencies are shown in Fig. 3. They have a different "radial" behavior, but a quasi-uniform electric field distribution in the outer part of the patch can be seen in both cases. These electric-field distributions produce the desired monopolar radiation pattern at both working frequencies (Fig. 4). The simulated gains are $2 \mathrm{dBi}$ at $2.6 \mathrm{GHz}$ and $2.7 \mathrm{dBi}$ at 4.8 $\mathrm{GHz}$. The electrical length of the patch is $\lambda_{0} / 3.72$ at the first frequency and $\lambda_{0} / 2.02$ at the second one. It is important to note that the electrical length of the patch is not increased with respect to the conventional patch antenna, but considerably reduced at the first working frequency. 


\section{References}

[1] V. González-Posadas, D. Segovia-Vargas, E. Rajo-Iglesias, J. L. Vázquez-Roy, and C. Martín-Pacual, "Approximate Analysis of Short Circuited Ring Patch Antenna Working at TM01 Mode", IEEE Transactions on Antennas and Propagation, vol. 54, no. 6, pp. 1875-1879, June 2006.

[2] N. Mercé, P. Tejedor, and J. Vassal'lo, "TheTM $\mathrm{T}_{01}$ mode of microstrip radiators and some of its applications," in IEEE Antennas and Propagation Society Int. Symp., pp. 33-36, Apr. 1995.

[3] C. Martín-Pascual and I. Seoane, "On the generation and applications of the monopolar ("zeroth" like) mode in non-circular patches," in Proc. 17th Int. Conf. on Applied Electromagnetics and Communications, Dubrovnik, Croatia, pp. 160-163, Oct. 2003.

[4] A. Lai, K. M. K. H. Leong, and T. Itoh, "Infinite Wavelength Resonant Antennas with Monopolar Radiation Pattern Based on Periodic Structures," IEEE Transactions on Antennas and Propagation, vol. 55, no. 3, pp. 868-876, March 2007.

[5] M. A. Antoniades and G. V. Eleftheriades, "A folded-monopole model for electrically small NRI-TL metamaterial antennas," IEEE Antennas Wireless Propag. Lett., vol. 7, pp. 425-428, 2008.

[6] G. Zhou, "Sorthing-Pin Loaded Annular Ring Microstrip Antenna," in IEEE Antennas and Propagation Society Int. Symp., vol. 2, pp. 33-36, Jun 1998.

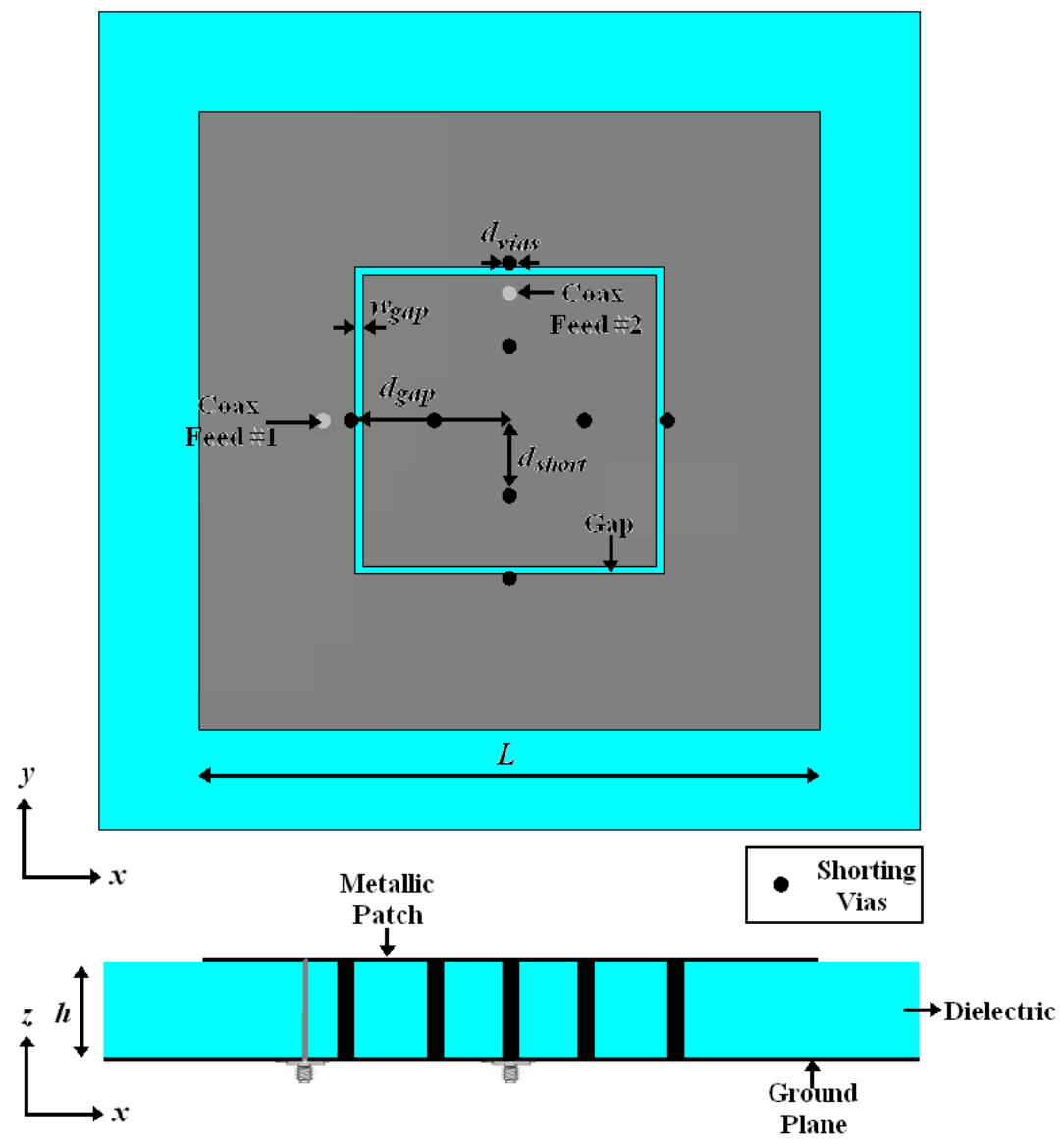

Fig. 1. Sketch of the proposed dual-frequency monopolar patch antenna. 


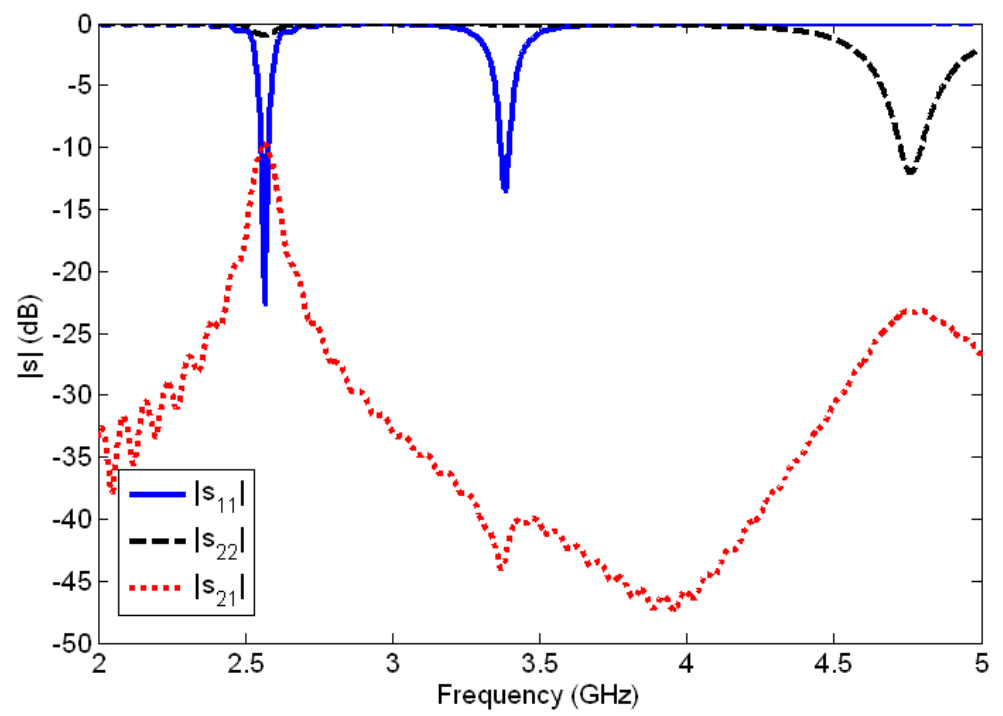

Fig. 2. Simulated $[\mathrm{S}]$ parameters of the proposed antenna.

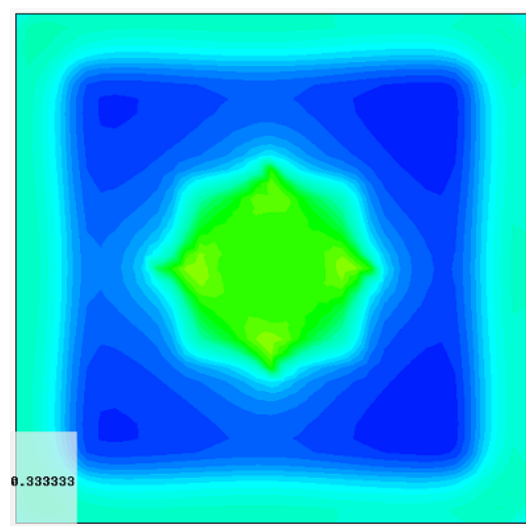

(a)
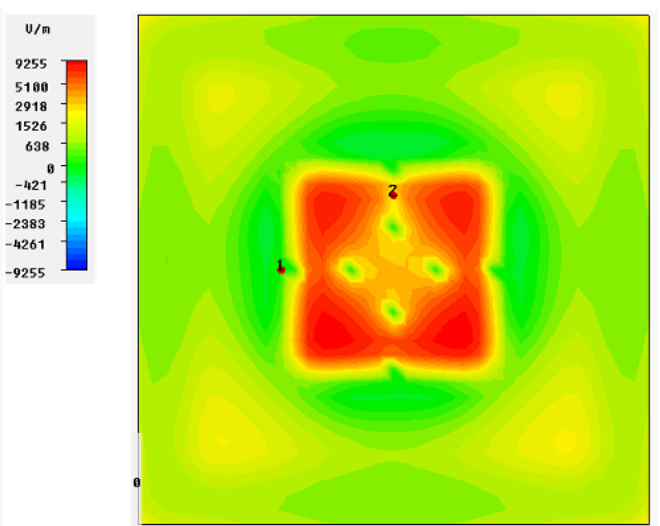

(b)

Fig. 3. Simulated electric-field distributions $\left(\mathrm{E}_{z}\right)$ of the proposed dual-frequency monopolar patch antenna. (a) $2.6 \mathrm{GHz}$. (b) $4.8 \mathrm{GHz}$.

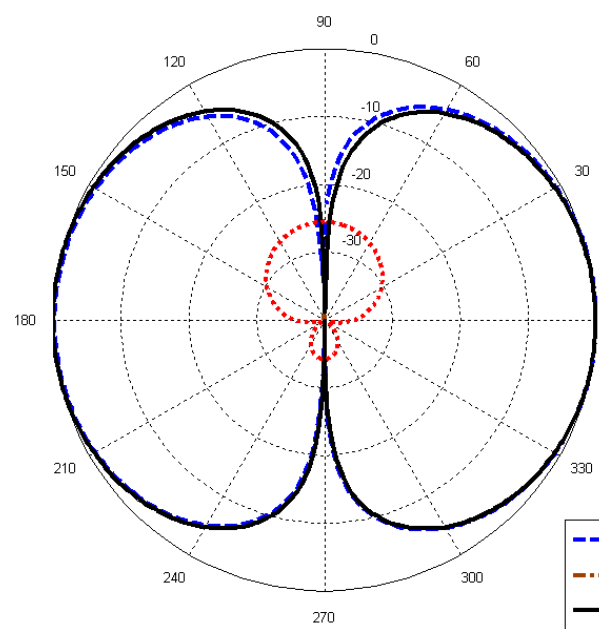

(a)

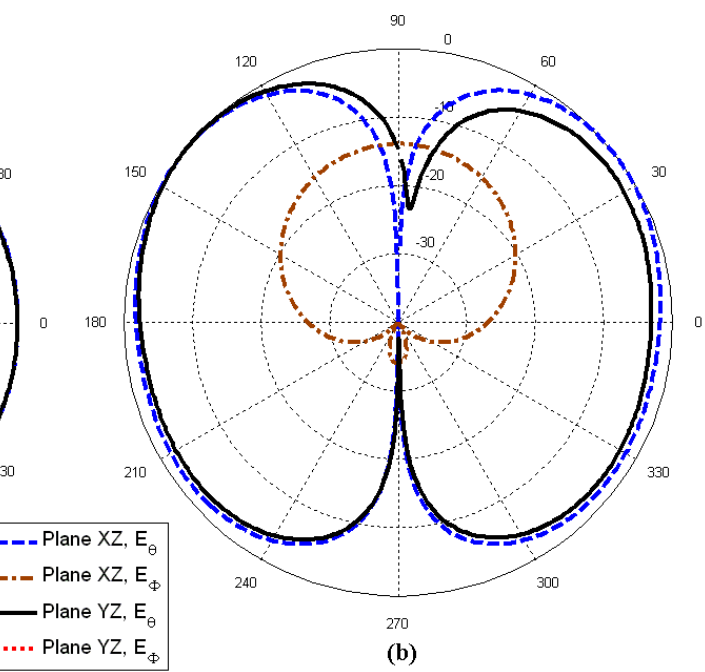

(b)

Fig. 3. Simulated radiation patterns of the proposed patch antenna. (a) $2.6 \mathrm{GHz}$. (b) 4.8 $\mathrm{GHz}$. 